\title{
Dynamic behavior analysis of Vibro-impact System with Two Motion Limited Constraints
}

\author{
Mei Lin \\ Air Force Engineering University Aeronautics and Astronautics Engineering College, Xi'an, Shaanxi, China
}

Guangshun Song

Xi'an Municipal Engineering Design \& Research Institute Co., Ltd, Xi'an, 710000, Shaanxi, China

Yongqiang Liu, Yanbo Zhu, Xiaolu Zhang

Air Force Engineering University Aeronautics and Astronautics Engineering College, Xi'an, Shaanxi, China

\begin{abstract}
Poincaré map is established for the dynamical model of a two-degree-of- freedom system with two constraints. Conditions of sticking are analyzed. Periodic motion is found by shooting method, namely fixed point of Poincaré section. Hopf bifurcation and period-doubling bifurcation are analyzed under suitable parameters by numerical simulation. As controlling parameter varies further, the route to chaos via quasi-period bifurcation and period-doubling bifurcation are investigated, respectively.
\end{abstract}

KEYWORD: vibro-impact; periodic motion; Poincaré map; sticking; bifurcation; chaos

\section{PREFACE}

In addition to the design clearance is required, operation factors may also cause clearance in many dynamical system. For the reason that clearance may cause collision in dynamical systems, studies on vibro-impact system with clearance attract more and more attention by many scholars abroad the world. Vibro-impact system is a complex nonlinear dynamic system, so studies on vibro-impact system with space impact have both important theoretical value and practical significance. Existed studies on vibro-impact system are mainly aimed at a single point of collision, seldom are aimed at more than two or two points of collision. The numerical simulation is performed for the transient response of a multiple-degree-of-freedom in paper I, Comparisons are made for the responses of the primary structure before and after attachment of a vibro-impact absorber to demonstrate its rapid mitigating effect on the vibration level as well as the robustness 。 A nonlinear dynamic model of two-degree- freedom dry friction oscillators with symmetric claranee was established. Slip, stick and impact of system motion were analyzed and their engaging conditions were proposed respectively in paper II. On the basis of mapping mechanism, some characteristic of dynamics of piecewise linear systems is studied in paper III.A three-degree-of-freedom vibro-impact system is studied in paper IV. Passing by quasi-periodic bifurcation and period doubling bifurcation, periodic motion changes to chaos when parameter of which changes. Evolutionary path of which is analyzed in this paper. Impact oscillator of two-degree-of- freedom changes when parameter of which changes. Viscous behavior and bifurcation type of which is studied in paper V. Uplift of vibro-impact system with two motion limiting constraints is studied in paper VI. By changing time interval of the system, shooting method for defining periodic orbit of the nonlinear system is submitted in paper VII. Complex dynamics problems of vibro-impact system with two motion limiting constraints and two-degree-of-freedom are studied in this paper. By setting up Poincaré map and using shooting method, fixed point of periodic motion in the system is obtained in this paper. Period doubling bifurcation and Hopf bifurcation of the system are also studied by numerical simulation.

\section{MECHANICAL MODEL OF TWO-DEGREE- OF- FREEDOM SYSTEM}

A dynamical model of two-degree-of- freedom system with gap is given in Fig.1. Two oscillators are joined to two linear dampers by two linear spcircles in this model. Mass of the two oscillators is $\mathrm{M}_{1}$ and $M_{2}$, stiffness of the two linear spcircles is $K_{1}$ and $K_{2}$, and damping coefficient of the two linear dampers are $\mathrm{C}_{1}$ and $\mathrm{C}_{2}$ respectively. The two oscillators move in horizontal direction only, acted upon displacement excitation valued $A \cos (\omega t+\tau)$. Oscillator $\mathrm{M}_{1}$ will collide with the rigid plane $\mathrm{B}_{1}$ and direction of which will change, starting a new motion with new initial value, when displacement $X_{1}$ of which equal to the gap S1.Being similar to M1, M2 will have the same action when displacement X2 of oscillator M2 equal to the gap S2. Then M1 and M2 will repeat the same action. It is assumed that the damping is a type of proportional damping named Rayleigh, expressed as $\mathrm{K}_{2} / \mathrm{K}_{1}=\mathrm{C}_{2} / \mathrm{C}_{1}$, and collision course depends on the coefficient of restitution in this model. The dimensionless differential equation of motion of the system in 
between any two successive collisions as the following,

$$
\begin{aligned}
& \ddot{x}_{1}+2 \zeta\left(1+\mu_{c}\right) \dot{x}_{1}-2 \zeta \mu_{C} \dot{x}_{2}+\left(1+\mu_{K}\right) x_{1}-\mu_{K} x_{2}=f_{10} \cos (\omega t+\tau) \\
& \mu_{m} \ddot{x}_{2}+2 \zeta \mu_{C} \dot{x}_{2}-2 \zeta \mu_{C} \dot{x}_{1}+\mu_{K} x_{2}-\mu_{K} x_{1}=f_{20} \cos (\omega t+\tau)
\end{aligned}
$$

In this equation, ' ${ }^{\prime \prime}$ is expressed as dimensionless time derivative. Specifying of the parameters is as the follow,

$$
\begin{aligned}
& \mu_{K}=\frac{K_{2}}{K_{1}}, \omega=\Omega \sqrt{\frac{M_{1}}{K_{1}}}, t=T \sqrt{\frac{K_{1}}{M_{1}}}, \\
& P_{1}=M_{1} \omega^{2} A \cos (\omega t+\tau), \zeta=\frac{C_{1}}{2 \sqrt{K_{1} M_{1}}} \\
& P_{2}=M_{2} \omega^{2} A \cos (\omega t+\tau), x_{i}=\frac{X_{i} K_{1}}{P_{1}+P_{2}} \\
& \mu_{C}=\mu_{K}=\frac{C_{2}}{C_{1}}=\frac{K_{2}}{K_{1}}, f_{20}=\frac{P_{2}}{P_{1}+P_{2}}, \\
& f_{10}=1-f_{20}, \quad s_{1}=\frac{S_{1} K_{1}}{P_{1}+P_{2}}, \quad s_{2}=\frac{S_{2} K_{1}}{P_{1}+P_{2}}
\end{aligned}
$$

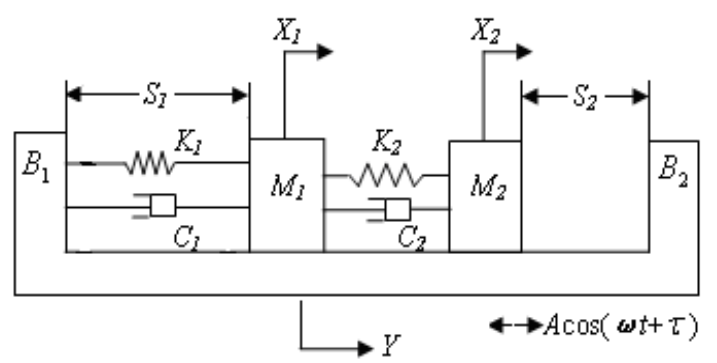

Fig.1 Mechanical Model of Two-degree -of-freedom System

The collision occurs when $x_{i}=s_{i}(i=1,2)$. Assumed that collision of $\mathrm{M}_{1}$ and $\mathrm{M}_{2}$ do not occur at the same time, and guidelines of coefficient of restitution is applied,

$$
\dot{x}_{i+}=-R \dot{x}_{i-} \quad(i=1,2)
$$

(2)

$\dot{x}_{i-}$ and $\dot{x}_{i+}(\mathrm{i}=1,2)$ stands for instantaneous velocity of $\mathrm{M}_{1}$ and $\mathrm{M}_{2}$ before and after collision in this equation. By mode superposition method, we can get solution of equation (1).

$x_{i}=\sum_{j=1}^{2} \psi_{i j}\left(e^{-\eta j t}\left(a_{j} \cos \left(\omega d_{j} t\right)+b_{j} \sin \left(\omega d_{j} t\right)\right)+A_{j} \sin (\omega t+\tau)+B_{j} \cos (\omega t+\tau)\right)$

$\psi_{i j}$ is an element of regular modal matrix $\psi$ in this equation, and $\eta_{j}=\zeta \omega_{j}^{2}, a_{j}, b_{j}$ are integral constants, identified by modal parameters and initial conditions of the system. $\omega_{d j}=\omega_{j} \sqrt{1-\zeta^{2}}, \omega_{1}, \omega_{2}$ stand for natural frequency of $\mathrm{M}_{1}$ and $\mathrm{M}_{2}$ without collision in the system. $A_{j}$ and $B_{j}$ are constant of amplitude.

\section{ANALYSIS OF THE VISCOUS MOTION}

Oscillator M1 or M2 will pause when they get in touch with plane B1 or B2. Such phenomenon is called viscous motion. Two types of viscous motion will exist in this system, they are $x_{1}=s_{1}, x_{2}=s_{2}$, assuming that $\mathrm{M}_{1}$ and $\mathrm{M}_{2}$ do not occur at the same time. If $x_{1}=s_{1}, \dot{x}_{1}=0$, resultant force F1 is negative direction, pointing to constraint, at the moment collision occurs between oscillator M1 and the constraint, oscillator M1 will stay at the constraint and viscous motion occurs. The system changes from two-degree-of-freedom to forced vibration with onedegree-of-freedom. Viscous motion will not finish until direction of F1 changes and a new motion with new initial value begins. Detention time as $t_{s 1}$, the equations of motion of the system in between any two successive collisions is as the follow,

$\mu_{m} \ddot{x}_{2}+2 \zeta \mu_{C} \dot{x}_{2}-2 \zeta \mu_{C} \dot{x}_{1}+\mu_{K} x_{2}-\mu_{K} x_{1}=f_{20} \cos (\omega t+\tau) \quad\left(0<t<t_{s 1}\right)$

When oscillator M1 has viscous motion, the force of which is,

$F_{1}=f_{10} \cos \left(\omega t_{1}+\tau\right)-2 \zeta\left(1+\mu_{c}\right) \dot{x}_{1}+2 \zeta \mu_{c} \hat{\dot{x}}_{2}-\left(1+\mu_{k}\right) x_{1}+\mu_{k} \hat{x}_{2}$

In this equation, $\hat{x}_{2}, \hat{\dot{x}}_{2}$ stand for displacement and velocity of oscillator $M_{2}$ respectively when viscous motion in oscillator $M_{1}$. When $0<t<t_{s 1}$, solution of equation (5) is as the follow,

$x_{2}=e^{-\eta_{2} t}\left(a_{2} \cos \left(\omega d_{2} t\right)+b_{2} \sin \left(\omega d_{2} t\right)+A_{2} \sin (\omega t+\tau)+B_{2} \cos (\omega t+\tau)+x_{0}\right.$

$A_{2}$ and $B_{2}$ are constant of amplitude and $x_{0}=s_{1}$ in this equation.

$a_{2}=x_{20}-A_{2} \sin (\tau)-B_{2} \cos (\tau)-x_{0}$

$b_{2}=\left(\dot{x}_{20}+\eta_{2}\left(x_{20}-x_{0}\right)-\left(B_{2} \eta_{2}+A_{2} \omega\right) \cos (\tau)-\left(A_{2} \eta_{2}-B_{2} \omega\right) \sin (\tau)\right) / \omega d_{2}$

In this equation, $x_{20}, \dot{x}_{20}$ stand for instant displacement and velocity of oscillator $M_{2}$ at the moment collision ends. Response charts of $M_{2}$ when viscous motion occurs is given in fig.2(a).Similarly, $\dot{x}_{2}=0, x_{2}=s_{2}$ when viscous motion occurs in $M_{2}$. Assume resistance time of $M_{2}$ is $t_{s 2}$. And the equations of motion of the system in between any two successive collisions is as the follow,

$\ddot{x}_{1}+2 \zeta\left(1+\mu_{c}\right) \dot{x}_{1}-2 \zeta \mu_{C} \dot{x}_{2}+\left(1+\mu_{K}\right) x_{1}-\mu_{K} x_{2}=f_{10} \cos (\omega t+\tau) \quad\left(0<t<t_{s 2}\right)$

When oscillator M2 has viscous motion, the force of which is,

$F_{2}=\left(f_{20} \cos (\omega t+\tau)-2 \zeta \mu_{c} \dot{x}_{2}+2 \zeta \mu_{c} \hat{\dot{x}}_{1}-\mu_{k} x_{2}+\mu_{k} \hat{x}_{1}\right) / \mu_{m}$

In this equation, $\hat{x}_{1}, \dot{x}_{1}$ stand for displacement and velocity of oscillator M1 respectively when viscous motion in oscillator $M_{2}$. Solution of equation (9) is as the follow,

$x_{1}=e^{-\eta_{1} t}\left(a_{1} \cos \left(\omega d_{1} t\right)+b_{1} \sin \left(\omega d_{1} t\right)+A_{1} \cos (\omega t+\tau)+B_{1} \cos (\omega t+\tau)+x_{h}\right.$

$A_{1}$ and $B_{1}$ are constant of amplitude and $x_{h}=\mu_{k} s_{2} /\left(1+\mu_{k}\right)$ in this equation.

$a_{1}=x_{10}-A_{1} \sin (\tau)-B_{1} \cos (\tau)-x_{h}$

$b_{1}=\left(\dot{x}_{10}+\eta_{1}\left(x_{10}-x_{h}\right)-\left(B_{1} \eta_{1}+A_{1} \omega\right) \cos (\tau)-\left(A_{1} \eta_{1}-B_{1} \omega\right) \sin (\tau)\right) / w d_{1}$ 
In this equation, $x_{10}, \dot{x}_{10}$ stand for instant displacement and velocity of oscillator $M_{2}$ when collision occurs.

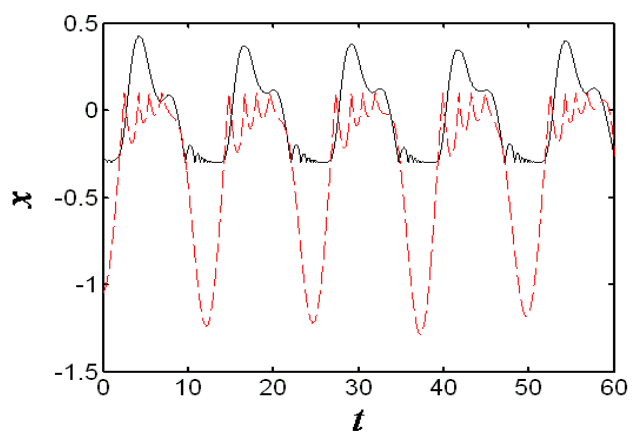

Fig.2 Response charts of two-degree-of- freedom vibro-impact system when viscous motion occurs

Solid line stands for $x_{1}$ and dashed line for $x_{2}$.Parameters are as the followings, $\mu_{k}=1, \mu_{m}=1$, $\zeta=0.05, s_{1}=-0.3, s_{2}=0.1, R=0.8, \omega=0.5, \quad f_{10}=0.5$, $f_{20}=0.5$.

\section{PERIODIC MOTION AND BIFURCATION OF VIBRO-IMPACT SYSTEM}

\subsection{Determination of Periodic Motion}

Make a section Poincaré,

$$
\begin{gathered}
\sigma=\left\{\left(x_{1}, \dot{x}_{1}, x_{2}, \dot{x}_{2}, \theta\right) \in R^{4} \times s \quad \mid x_{1}=s_{1}, \dot{x}_{1}=\dot{x}_{1+}\right\} \\
\theta=\omega t
\end{gathered}
$$

We know, by numerical solution, stable fixed point (stable periodic motion) in section Poincaré can be close to indefinitely, but instable fixed point can't.Periodic motion of the system can be expressed as $n-p-q$. In this expression, $\mathrm{n}$ stands for number of periodicity, $\mathrm{p}$ stands for collision times happened between oscillator $\mathrm{M}_{1}$ and constraint $\mathrm{B}_{1}$, q stands for collision times happened between oscillator $\mathrm{M}_{2}$ and constraint $\mathrm{B}_{2}$.Shooting method is used to get instable periodic motion and get fixed point in section Poincaré.In iterative process, it can be regarded as periodic fixed point when distance of the two adjacent points is less than a specified precision $\varepsilon$. The upper process can be described as follow,

Firstly, a group of initial value is selected for iteration, the iteration times of $\mathrm{i}$ is,

$$
\left(x_{2}(i+1), \dot{x}_{1}(i+1), \dot{x}_{2}(i+1), \tau(i+1)\right)=f\left(x_{2}(i), \dot{x}_{1}(i), \dot{x}_{2}(i), \tau(i)\right)
$$

Assume $\mathrm{h}$ is stride length of the iteration, adding a stride length to $x_{2}(i)$, the equation change to,

$\left(x_{2+h}(i+1), \dot{x}_{1+h}(i+1), \dot{x}_{2+h}(i+1), \tau_{+h}(i+1)\right)=f\left(x_{2}(i)+h, \dot{x}_{1}(i), \dot{x}_{2}(i), \tau(i)\right)$

Subtract a stride length to $x_{2}(i)$, the equation change to,

$$
\left(x_{2-h}(i+1), \dot{x}_{1-h}(i+1), \dot{x}_{2-h}(i+1), \tau_{-h}(i+1)\right)=f\left(x_{2}(i)-h, \dot{x}_{1}(i), \dot{x}_{2}(i), \tau(i)\right)
$$

For the iteration times of I, induced norm can be defined as,

$$
\begin{aligned}
& \|d(i)\|=\left(\left(x_{2}(i+1)-x_{2}(i)\right)^{2}+\left(\dot{x}_{2}(i+1)-\dot{x}_{2}(i)\right)^{2}\right)^{\frac{1}{2}} \\
& \left\|d_{+h}(i)\right\|=\left(\left(x_{2}(i+1)-\left(x_{2}(i)+h\right)\right)^{2}+\left(\dot{x}_{2}(i+1)-\dot{x}_{2}(i)\right)^{2}\right)^{\frac{1}{2}} \\
& \left\|d_{-h}(i)\right\|=\left(\left(x_{2}(i+1)-\left(x_{2}(i)-h\right)\right)^{2}+\left(\dot{x}_{2}(i+1)-\dot{x}_{2}(i)\right)^{2}\right)^{\frac{1}{2}}
\end{aligned}
$$

Assume $\varepsilon$ as a specified precision, if the following conditions are true,

$\lim _{h \rightarrow 0}\|d(i)\|<\varepsilon$,

$\lim _{h \rightarrow 0}\left\|d_{-h}(i)\right\|<\varepsilon$,

$\lim _{h \rightarrow 0}\left\|d_{+h}(i)\right\|<\varepsilon$,

$x_{2}(i)$ can be regarded as the solution, which can be expressed as $\hat{x}_{20}=x_{2}(i)$.

If $\left\|d_{+h}(i)\right\|<\|d(i)\|<\left\|d_{-h}(i)\right\|$, then add a stride length to $x_{2}(i)$, $x_{2}(i)=x_{2}(i)+h$

If $\left\|d_{+h}(i)\right\|>\|d(i)\|>\left\|d_{-h}(i)\right\|$, then subtract a stride length to $x_{2}(i)$,

$x_{2}(i)=x_{2}(i)-h$

If $\left\|d_{+h}(i)\right\|>\|d(i)\|$ and $\|d(i)\|<\left\|d_{-h}(i)\right\|$, then add a half stride length to $x_{2}(i)$,

$x_{2}(i)=x_{2}(i), h=h / 2$

Plug $x_{2}(i)$ into equation 14,15 and 16, and have another iterative process until equation 20is true.

Respectively, we can get

$\hat{\dot{x}}_{10}=\dot{x}_{1}(i), \dot{x}_{20}=\dot{x}_{2}(i), \hat{\tau}_{0}=\tau(i) \quad$ by iterative calculation.

$\hat{x}_{20}, \hat{\dot{x}}_{10}, \hat{\dot{x}}_{20}, \hat{\tau}_{0}$ is not accurate enough. Take $\hat{x}_{20}, \dot{x}_{10}, \dot{x}_{20}, \hat{\tau}_{0}$ as initial mapping points and decrease the number of precision $\varepsilon$, having new iterative calculations, we can get periodic fixed point in section Poincaré. 。

Evolution process of getting periodic fixed point is represented in figure 3.

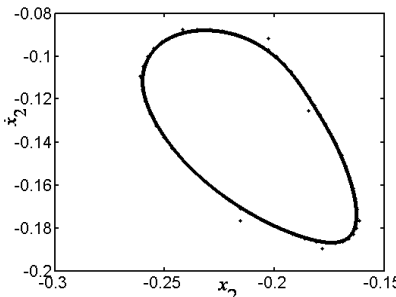

(a)

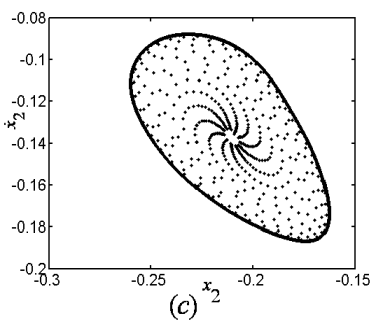

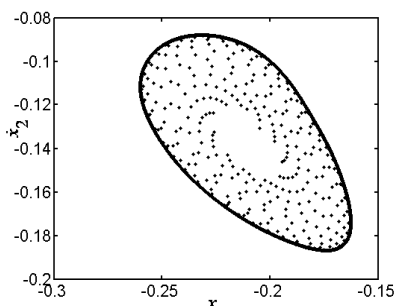

$(b)^{x_{2}}$

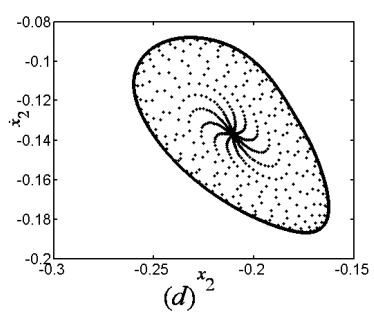

Fig. 3 Evolution Process of Periodic Fixed Point 


\subsection{Chaos and Bifurcation of Periodic Motion}

One group of parameter of vibro-impact system as $\mu_{m}=1, \quad \mu_{k}=1, R=0.8, \zeta=0.012, \quad s_{1}=-0.3$, $s_{2}=0.1, f_{10}=0.5, f_{20}=0.5$ is taken in fig. 3 .

Taken $\omega$ as bifurcation parameter of the system, part bifurcation diagram of the system is given in fig. 4. In fig. 2, the system changes from quasi-periodic motion to bifurcation of periodic motion. One periodic motion appears when $\omega \in(1.29-1.302)$, and doubling periodic motion appears when $\omega \in(1.565-$ 1.625). The system gets into chaos gradually when $\omega$ arises, showing how the system changes from periodic bifurcation to chaos. Another group of parameter of vibro-impact system as $\mu_{m}=1, \mu_{k}=5$, $R=0.8, \quad \zeta=0.05, \quad s_{1}=-0.2 \quad, \quad s_{2}=0.1$, $f_{10}=0.5$, and $f_{20}=0.5$ is taken in fig. 1 .

Overseeing dynamics behaviors show in fig. 5 . Doubling periodic motion appears in the system when $\omega \in(1.65-1.83)$ and the system gets into chaos when the parameter $\omega$ arises. Periodic motion and bifurcation appears in the system when bifurcation parameter is $\omega \in(2.1-2.8)$, no double sequence appears now. The system will change from periodic motion to quasi-periodic motion and then to chaos when the parameter $\omega$ arises. The system will out of chaos and quasi-periodic motion will appear again with the further increase of control parameters, ending in one stable periodic motion. The process shows how the system changes from quasi-periodic motion to chaos. Numerical simulation results reveal part and overseeing dynamic characteristic of the flexibleplastic vibro-impact system, showing complex dynamic behavior of Vibro-impact system with two motion limited constraints and the way the system changes to chaos.

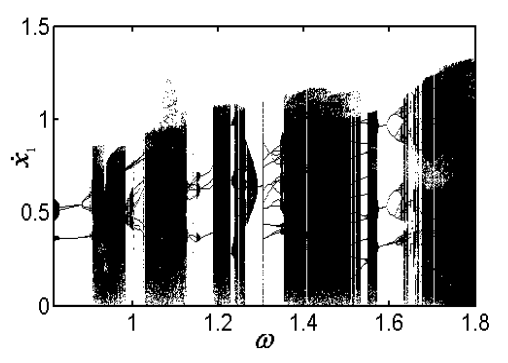

Fig. 4 local bifurcation

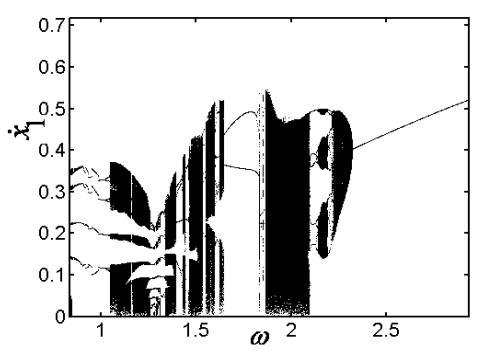

Fig. 5 global bifurcation

\section{CONCLUSIONS}

Vibro-impact system with two motion limiting constraints and two-degree-of- freedom and Poincaré mapping are built in this paper. Requirement that viscous motion occurs in the system is analyzed, trying to resolve viscous motion in the system.Using shooting method and multi-iteration by changing stride length, we get fixed point in section Poincaré.

Bifurcation and chaos of periodic motion of in the system is analysed in this paper, revealing two ways how the system changes to chaos, which are from periodic bifurcation and quasi-periodic bifurcation. Complex dynamic behavior of Vibro-impact system with two motion limited constraints is also revealed in this paper.

\section{REFERENCE}

Albert C. J. Luo, Lidi chen. Periodic motions and grazing in a harmonically forced, piecewise, linear oscillator with impacts. Chaos, Solitons \& Fractals, 2005,24(2):567-578.

Ding WC, Xie J H,li GF. Stability and bifurcations of periodic motion in athree-degree-of-freedom vibro-impact system. Engineering dynamics, 2004,24(3): 123-128

D. J. Wagg. Periodic sticking motion in a two-degree-of- freedom impact oscillator. Int. J. Non-Linear Mech, 2005, 40(8): 1076-1087.

D. J. Wagg. Rising phenomena and the multi-sliding bifurcation in a two-degree of freedom impact oscillator. Chaos, Solitons \& Fractals, 2004, 22(3): 541-548.

Li DX, Xiu JX Generalized shotting method for determining periodic orbit of the nonlinear dynamical system Chinese Journal of applied mechanics, 2003(4): 80-85

le Y, Xie J H,Ding WC.Hopf bifurcation and chaos of a twodegree-of- freedom Vibro-impact system. Journal of dynamics and control, 2004, 2(3): 36-41

Wang Dong. Analysis of Vibration Energy Dissipation with Vibro-impact Absorber,Journal of Mechanical Engineering,2014,17: 88-92. 